\title{
Power-Interrogated Refractive Index Sensor Using Long Period Grating in Photonic Crystal Fiber
}

\author{
X. L. Jiang, ${ }^{1,2}$ Z. T. Gu, ${ }^{1}$ and L. Zheng ${ }^{2}$ \\ ${ }^{1}$ College of Science, University of Shanghai for Science and Technology, Shanghai 200093, China \\ ${ }^{2}$ Department of Math and Physics, Shanghai Institute of Electric Power, Shanghai 200090, China \\ Correspondence should be addressed to X. L. Jiang; jiangxiuli@shiep.edu.cn and Z. T. Gu; zhengtiangu@163.com
}

Received 2 February 2015; Accepted 11 March 2015

Academic Editor: Ruomeng Yu

Copyright (c) $2015 \mathrm{X}$. L. Jiang et al. This is an open access article distributed under the Creative Commons Attribution License, which permits unrestricted use, distribution, and reproduction in any medium, provided the original work is properly cited.

\begin{abstract}
We reported a long period grating (LPG) written in specially designed photonic crystal fiber (PCF) for refractive index (RI) sensing by interrogating the transmitted light power. The outermost ring of clad holes of the PCF is enlarged where the analyte is filled. We showed that the leakage loss of the clad mode increases with the RI in the larger holes. By numerically analyzing the complex couple mode equations for the core mode and the first clad mode, we found the depth of attenuation band in the transmitted spectra and the total transmitted power is sensitive to the leakage loss of the clad mode or the RI in the larger holes. We also demonstrated that the transmitted power is sensitive to the RI even less than that of the silica, which just avoids the limitation that the transmitted light power of LPG in conventional fiber is only sensitive to the RI of the external media higher than that of fiber clad.
\end{abstract}

\section{Introduction}

The introduction of periodic structure in optical fiber has been used in many optical fields. Long period fiber grating (LPFG) can couple the core mode to the cladding modes which are sensitive to the refractive index (RI) of the surrounding medium. A number of studies on the RI sensitivity of LPFGs have been conducted [1-4]. A study on RI sensing using LPFGs by Patrick et al. has demonstrated that a change in RI lower than the index of fiber clad causes wavelength shift, while a change in RI higher than the index of the fiber clad causes changes in shape and magnitude of attenuation bands [5]. Many experiments demonstrated that a long period grating in a photonic crystal fiber (PCF-LPG) can be used for RI sensing, in which a much smaller sample volume is possible by using the holes in the cladding as microfluidic channels [6-8]. The previous RI sensors using PCF-LPGs monitored the changes in RI by the shift of the resonant wavelength of the PCF-LPGs. However, one intrinsic problem of such sensors is their cross sensitivity to other parameters especially to the temperature; another practical disadvantage is complex wavelength-dispersive demodulator which is necessary.
This letter reports an LPG written in specially designed photonic crystal fiber, and its magnitude of the attenuation bands varies with the RI even lower than the fiber clad and so does the total transmitted light power. With this sensing mechanism, only an optical power meter is required for signal interrogation. The transverse geometry of the PCF and the computation model are depicted in Figure 1. The cross section of the fiber includes the following parts: the Ge-doped core, the clad consisting of five rings of air holes, the fifth ring of enlarged holes through which the analyte is transmitted, and the outermost silica region truncated by the perfectly matched layer (PML) [9].

The clad modes of PCF are leaky as some light can leak out of the cladding with finite rings of inclusions through the channels between every two of the inclusions. The leakage can be easily manipulated by changing the air hole structure or the RI of the medium in the holes $[10,11]$. Here we numerically demonstrate that the leakage loss of the clad mode increases with the RI of the medium in the larger holes, which leads to the reduction of the coupling strength between the core mode and the clad mode and the changes in the magnitude of the attenuation band. The analyte is filled in the holes of the outermost layer, leaving others unfilled. There are 


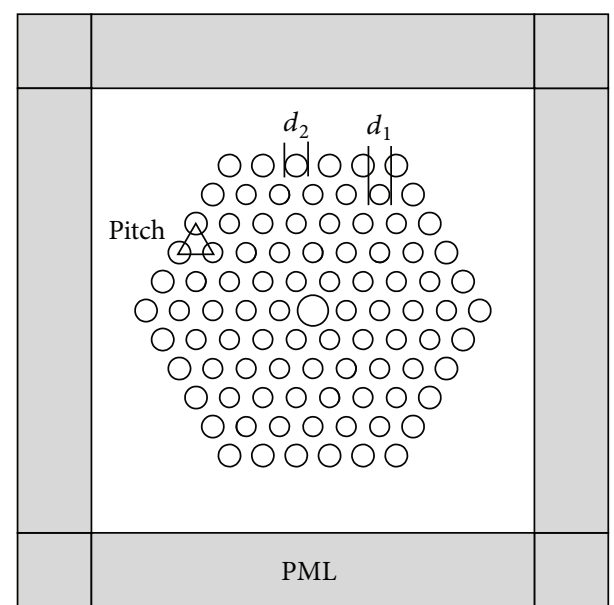

FIGURE 1: The transverse cross section of the PCF truncated by PML.

a number of techniques for achieving selective filling, including collapsing air holes and direct manual gluing [12].

\section{Theory}

The transmission characteristics of an LPG-PCF can be investigated by the complex coupled mode theory [13-15]. To illustrate the leakage loss of the $m$ th clad mode in the PCF, an imaginary term should be introduced into the mode propagation constant $\beta_{\mathrm{cl}, m}=\beta^{\prime}+i \alpha$, so the complex couple mode equation for the core mode and the $m$ th clad mode can be expressed as

$$
\begin{aligned}
& \frac{d A_{\mathrm{co}}}{d z}=i K A_{\mathrm{cl}, m} \exp (-\alpha z) \times \exp \left[-i\left(\beta_{\mathrm{co}}-\beta^{\prime}-\frac{2 \pi}{\Lambda}\right) z\right], \\
& \frac{d A_{\mathrm{cl}, m}}{d z}=i K A_{\mathrm{co}} \exp (\alpha z) \times \exp \left[-i\left(\beta_{\mathrm{co}}-\beta^{\prime}-\frac{2 \pi}{\Lambda}\right) z\right] .
\end{aligned}
$$

$A_{\mathrm{co}}$ and $A_{\mathrm{cl}, m}$ are the amplitudes of the core mode and the $m$ th clad mode, respectively. $\beta_{\mathrm{co}}$ is the propagation constant of the core mode, $\Lambda$ is the grating period, $K$ is the coupling coefficient which is proportional to the average refractive index modulation and overlap integral between the two coupled modes.

When the phase matching condition $\beta_{\mathrm{co}}-\beta^{\prime}-2 \pi / \Lambda=0$ is satisfied, the couple mode equation can be rewritten as

$$
\begin{aligned}
& \frac{d A_{\mathrm{co}}}{d z}=i K A_{\mathrm{cl}, m} \exp (-\alpha z), \\
& \frac{d A_{\mathrm{cl}, m}}{d z}=i K A_{\mathrm{co}} \exp (\alpha z) .
\end{aligned}
$$

Equation about $A_{\text {co }}$ can be deduced from (2):

$$
\frac{d^{2} A_{\mathrm{co}}}{d z^{2}}+\alpha \frac{d A_{\mathrm{co}}}{d z}+K^{2} A_{\mathrm{co}}=0
$$

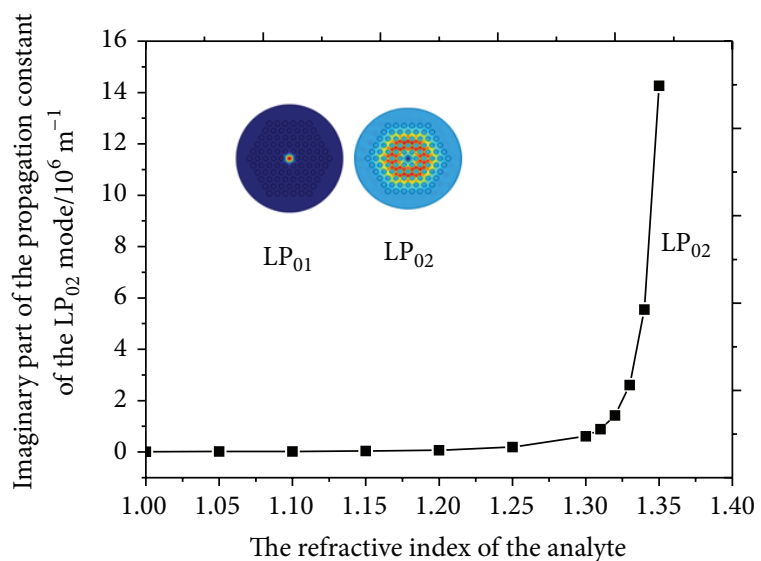

FIGURE 2: Effect of RI of the analyte on the imaginary part of propagation constant of the clad mode $\mathrm{LP}_{02}$.

The amplitude of the core mode is just like a damped oscillator. The transmission at the resonant wavelength of the LPG with length of $L$ can be got from (3):

$$
\begin{gathered}
I=\frac{A_{\mathrm{co}}{ }^{2}(L)}{A_{\mathrm{co}}{ }^{2}(0)}=e^{-\alpha L} \cos ^{2}\left(\sqrt{K^{2}-\left(\frac{\alpha}{2}\right)^{2}} L-\varphi\right), \\
\varphi=\tan ^{-1}\left(\frac{\alpha / 2}{\sqrt{K^{2}-(\alpha / 2)^{2}}}\right) .
\end{gathered}
$$

\section{Numerical Results}

An efficient coupling between fundamental core modes $\mathrm{LP}_{01}$ and $\mathrm{LP}_{0 n}$ group of clad modes that have similar electric field profiles and large overlap integrals will take place by periodic perturbation along the fiber. The mode properties of the core mode $\mathrm{LP}_{01}$ and the lowest-order clad mode $\mathrm{LP}_{02}$ and the coupling between the two modes have been theoretically investigated. The electric fields of the two modes are depicted in the inset of Figure 2. In the simulation, the refractive index of silica is obtained from Sellmeier formula, the refractive index of the Ge-doped core is 0.012 higher than that of the silica, and the diameter of the doped core is $4.14 \mu \mathrm{m}$. The diameter of the inner four layers of air holes is $d_{1}=2.65 \mu \mathrm{m}$, the diameter of the fifth layer holes is $d_{2}=3 \mu \mathrm{m}$, and the hole pitch is $4.52 \mu \mathrm{m}$.

The finite element method (FEM) incorporating anisotropic perfectly matched layers (PML) as absorbing boundary conditions allows us to evaluate the effective index of the leaky mode in PCFs including the real and imaginary part. The imaginary part of the propagation constant of the core mode equals zero due to the fact that the doped core has higher RI. The imaginary part of the propagation constant of clad mode $\mathrm{LP}_{02}$ versus the RI of the analyte in the larger holes is plotted and shown in Figure 2. With the outermost air holes enlarged, the imaginary part of the propagation constant of mode $\mathrm{LP}_{02}$ is negligible which is decreased by $\sim 3$ orders of magnitude compared to without enlarging the holes. The clad 


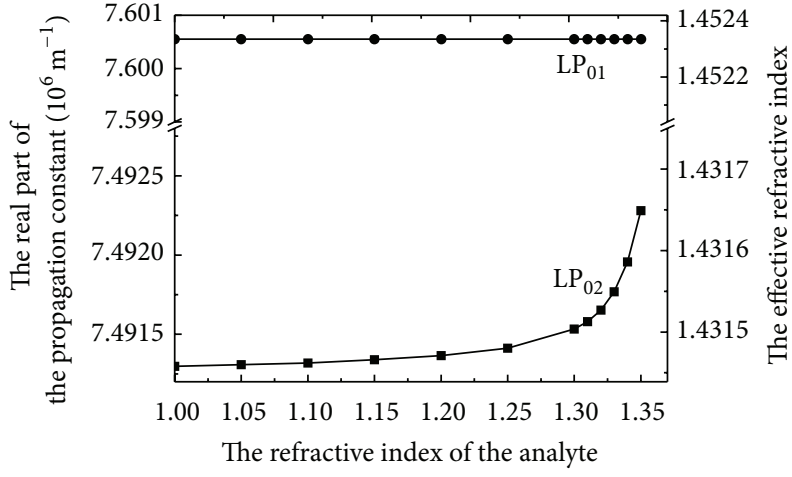

FIGURE 3: Effect of RI of the analyte on the real parts of propagation constant of the two modes: $\mathrm{LP}_{01}$ and $\mathrm{LP}_{02}$.

mode is well confined by the enlarged air holes. As the RI of the analyte increases, the imaginary part $(\alpha)$ of the propagation constant of $\mathrm{LP}_{02}$ mode increases. The imaginary part of the propagation constant of $\mathrm{LP}_{02}$ increases drastically when the RI of the analyte exceeds 1.3 and increases to the same order of magnitude of $K$ at the RI of 1.33. (The grating is designed to have the coupling coefficient $K$ of $40 / \mathrm{m}^{-1}$ and coupling strength $K L$ of $\pi / 2$ for air in all the holes.) According to (3), the bigger the $\alpha / 2$ is, the slower the $A_{\text {co }}$ reaches zero. For certain grating length of $L$, obvious increase in the transmission at the resonant wavelength can be achieved due to the dramatic growth of the imaginary part of the clad mode when the RI of the analyte exceeds 1.3 .

Figure 3 shows the effect of the refractive index of the analyte in the larger holes on the real parts of the two modes propagation constants. The wavelength is $1200 \mathrm{~nm}$. It can be seen from Figure 3 that the core mode is not affected by the RI of the analyte, and the real part of the propagation constant of the $\mathrm{LP}_{02}$ mode increases as the $\mathrm{RI}$ of the analyte increases, which will cause the blue shift of the resonant wavelength.

The shift of the resonant wavelength caused by the changes of the RI in the larger holes has been calculated by the following steps. Firstly, the real parts of the effective refractive index of $\mathrm{LP}_{01}$ and $\mathrm{LP}_{02}$ at different wavelength for certain $\mathrm{RI}$ in the larger holes are calculated, and then a set of periodicities that meet the phase-matching condition $\lambda=\left(n_{\mathrm{LP}_{01}}-\right.$ $\left.n_{\mathrm{LP}_{02}}\right) \Lambda$ at given wavelengths are obtained. In Figure 4 we have plotted the calculated grating period versus the resonant wavelength for RI of $1,1.33$, and 1.34 in the larger holes. For the LPG with the period of $57.48 \mu \mathrm{m}$, the resonant wavelength is $1200 \mathrm{~nm}, 1176 \mathrm{~nm}$, and $1169 \mathrm{~nm}$, respectively. The resonant wavelength shifts $7 \mathrm{~nm}$ from $1176 \mathrm{~nm}$ to $1169 \mathrm{~nm}$ when the RI in the larger holes changed from 1.33 to 1.34 .

To study the response of the transmission spectra to the RI in the larger holes, we calculated the transmission spectra by integrating numerically the coupled-mode-equations (1) for the RI of $1,1.33$, and 1.34, respectively. The calculated spectra are plotted in Figure 5. The position of the resonant wavelength is consistent with the result of the previous method which manifests the resonant wavelength depending on the real part of the propagation constant of the two modes. The LPG is designed to completely couple the core mode to

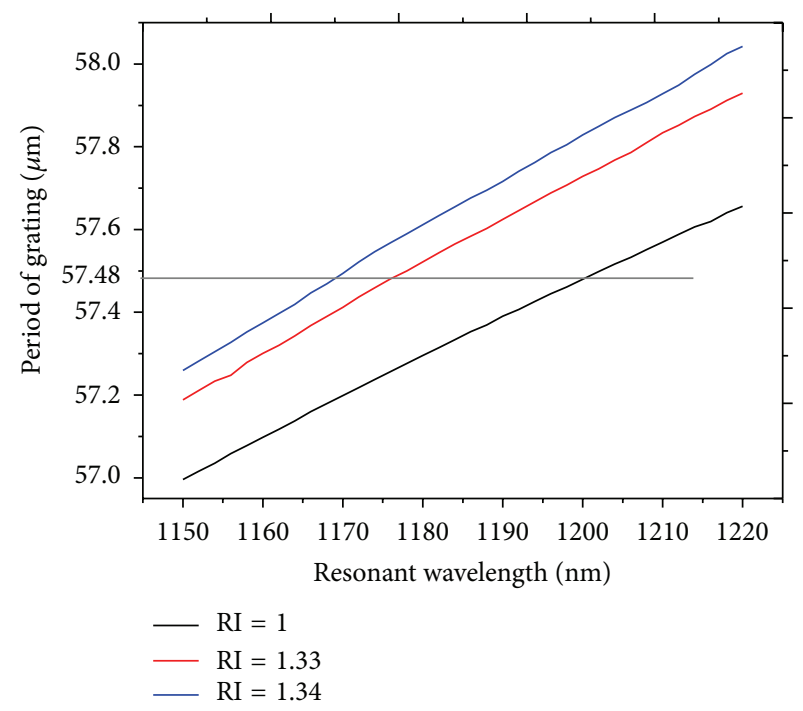

FIGURE 4: Relationship between grating periodicity and resonance wavelength.

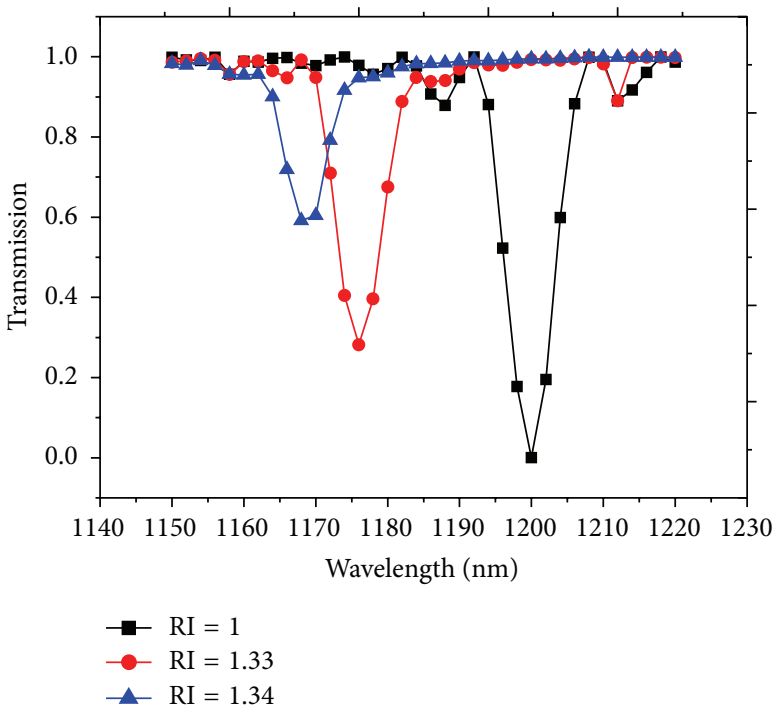

FIgURE 5: Transmission spectra for different RI in the enlarged holes.

the clad mode for the RI is 1 (air in the larger holes); hence the transmission at the resonant wavelength is equal to zero. When the RI in the larger holes increases from 1.33 to 1.34 , the depth of the attenuation band decreases obviously; meanwhile the whole transmitted light power increases. Such variation is mainly caused by the increase in the imaginary part $(\alpha)$ of the propagation constant of $\mathrm{LP}_{02}$.

Supposing a broadband LD light source with spectra range of $1160 \mathrm{~nm}-1184 \mathrm{~nm}$ and the spectral power of $0.1 \mathrm{mw} /$ $\mathrm{nm}$, the integrated transmitted power over the range of $1160 \mathrm{~nm}-1184 \mathrm{~nm}$ would be $1.83 \mathrm{mw}$ and $2.06 \mathrm{mw}$ for RI of 1.33 and 1.34 , respectively, which indicates the possibility using LPG based on the proposed PCF as RI sensor by interrogating the transmitted light power. The less the attenuation band shifts, the narrower the incident spectral range can be chosen 
and the greater the contrast the output light power has, that is, the more sensitive the sensor is. The shift of the resonant wavelength can be minimized by optimizing the air hole structure of the PCF.

\section{Conclusion}

In conclusion, we have demonstrated the feasibility of LPGPCF RI sensor by interrogating the transmitted light power in this paper. The transmitted light power is sensitive to the RI less than that of the silica, which just avoided the limitation that the transmitted light of LPG in conventional fiber is only sensitive to the RI of the external media higher than that of fiber clad.

\section{Conflict of Interests}

The authors declare that there is no conflict of interests regarding the publication of this paper.

\section{Acknowledgments}

This work is jointly supported by National Science Foundation of China (Grant no. 60777035), National Science Foundation of China (Grant no. 11204172), Innovation Program of Shanghai Municipal Education (no. 13YZ104), Shanghai Leading Academic Discipline Project (no. S30502), and Innovation Project of Shanghai Municipal Education Committee (no. 11ZZ131).

\section{References}

[1] Z. Gu, Y. Xu, and K. Gao, "Optical fiber long-period grating with solgel coating for gas sensor," Optics Letters, vol. 31, no. 16, pp. 2405-2407, 2006.

[2] X. L. Jiang and Z. T. Gu, "Design of a gas sensor based on a sensitive film coated phase-shifted long-period fiber grating," Journal of Optics, vol. 12, no. 7, Article ID 075401, 2010.

[3] S. W. James and R. P. Tatam, "Optical fibre long-period grating sensors: characteristics and application," Measurement Science and Technology, vol. 14, no. 5, pp. R49-R61, 2003.

[4] L. Zheng, X. Chen, S. Tang, and R. Li, "Multiple quasi-phasematching for enhanced generation of selected high harmonics in aperiodic modulated fibers," Optics Express, vol. 15, no. 26, pp. 17985-17990, 2007.

[5] H. J. Patrick, A. D. Kersey, and F. Bucholtz, "Analysis of the response of long period fiber gratings to external index of refraction," Journal of Lightwave Technology, vol. 16, no. 9, pp. 1606-1612, 1998.

[6] L. Rindorf, J. B. Jensen, M. Dufva, P. E. Høiby, L. H. Pedersen, and O. Bang, "Photonic crystal fiber long-period gratings for biochemical sensing," Optics Express, vol. 14, no. 18, pp. 82248231, 2006.

[7] Z. He, Y. Zhu, and H. Du, "Long-period gratings inscribed in air- and water-filled photonic crystal fiber for refractometric sensing of aqueous solution," Applied Physics Letters, vol. 92, no. 4, Article ID 044105, 2008.

[8] L. Rindorf and O. Bang, "Highly sensitive refractometer with a photonic-crystal-fiber long-period grating," Optics Letters, vol. 33, no. 6, pp. 563-565, 2008.
[9] C.-H. Lai and H.-C. Chang, "Effect of perfectly matched layer reflection coefficient on modal analysis of leaky waveguide modes," Optics Express, vol. 19, no. 2, pp. 562-569, 2011.

[10] T. P. White, R. C. McPhedran, C. M. De Sterke, L. C. Botten, and M. J. Steel, "Confinement losses in microstructured optical fibers," Optics Letters, vol. 26, no. 21, pp. 1660-1662, 2001.

[11] Z. H. He, Y. A. Zhu, J. Kaňka, and H. Du, "Core-cladding mode coupling and recoupling in photonic crystal fiber for enhanced overlap of evanescent field using long-period gratings," Optics Express, vol. 18, no. 2, pp. 507-512, 2010.

[12] B. T. Kuhlmey, B. J. Eggleton, and D. K. C. Wu, "Fluid-filled solid-core photonic bandgap fibers," Journal of Lightwave Technology, vol. 27, no. 11, pp. 1617-1630, 2009.

[13] D. B. Stegall and T. Erdogan, "Leaky cladding mode propagation in long-period fiber grating devices," IEEE Photonics Technology Letters, vol. 11, no. 3, pp. 343-345, 1999.

[14] X. Daxhelet and M. Kulishov, "Theory and practice of longperiod gratings: when a loss becomes a gain," Optics Letters, vol. 28, no. 9, pp. 686-688, 2003.

[15] X. L. Jiang and Z. T. Gu, "Impact of thin film extinction coefficient on coated long-period fiber grating transmission spectrum," Acta Optica Sinica, vol. 30, no. 8, pp. 2189-2195, 2010. 

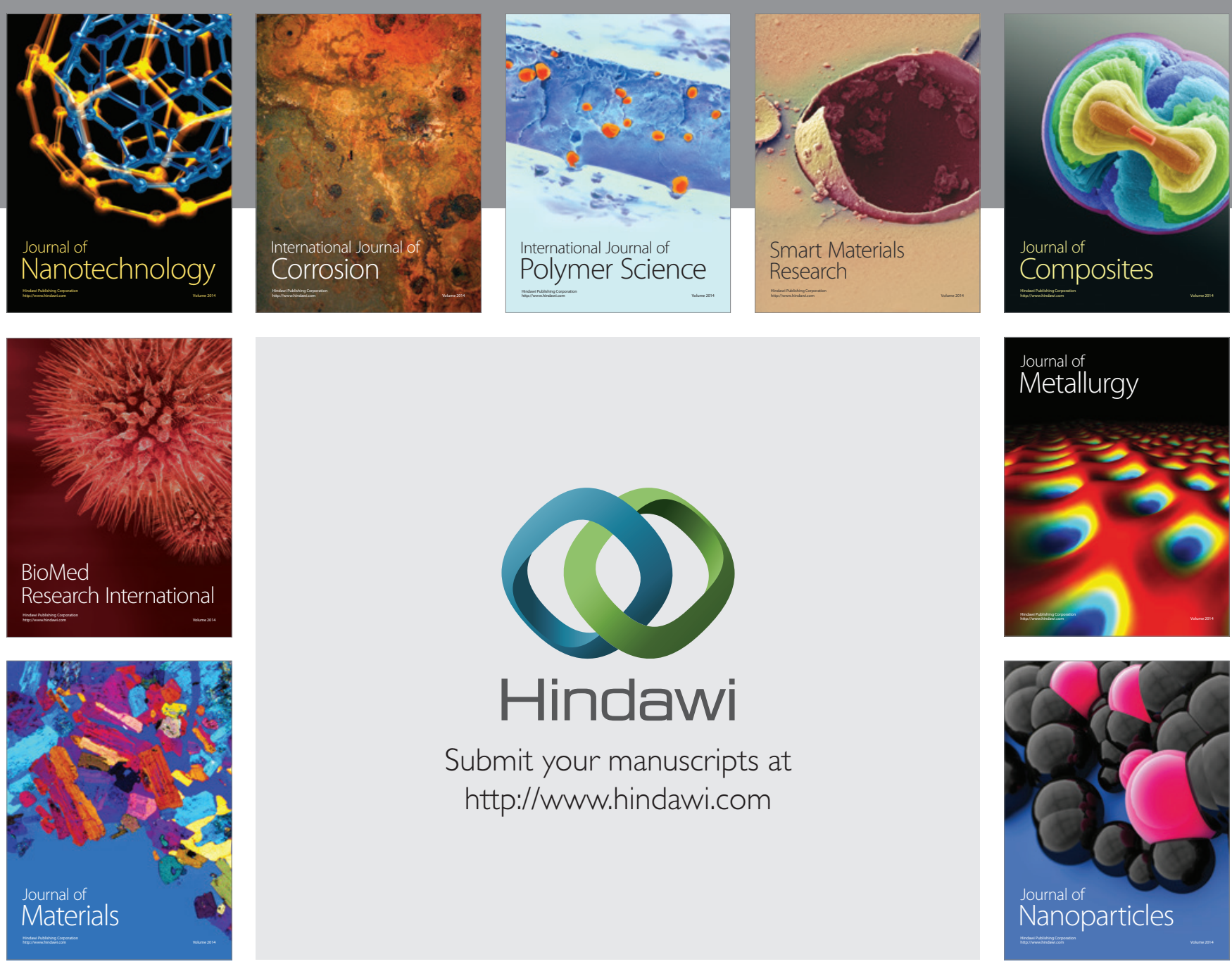

Submit your manuscripts at http://www.hindawi.com
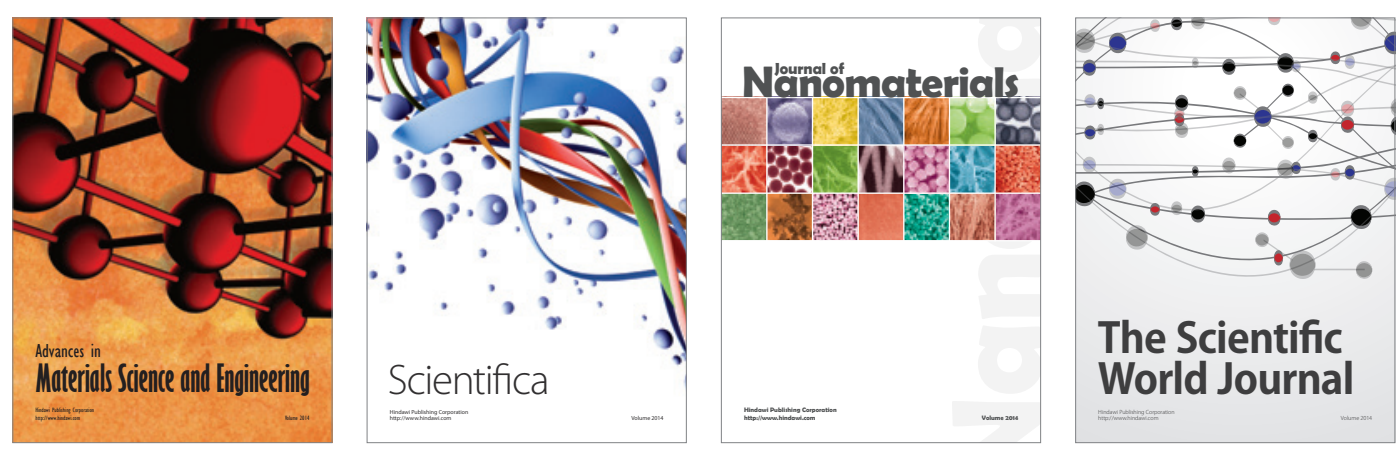

\section{The Scientific World Journal}
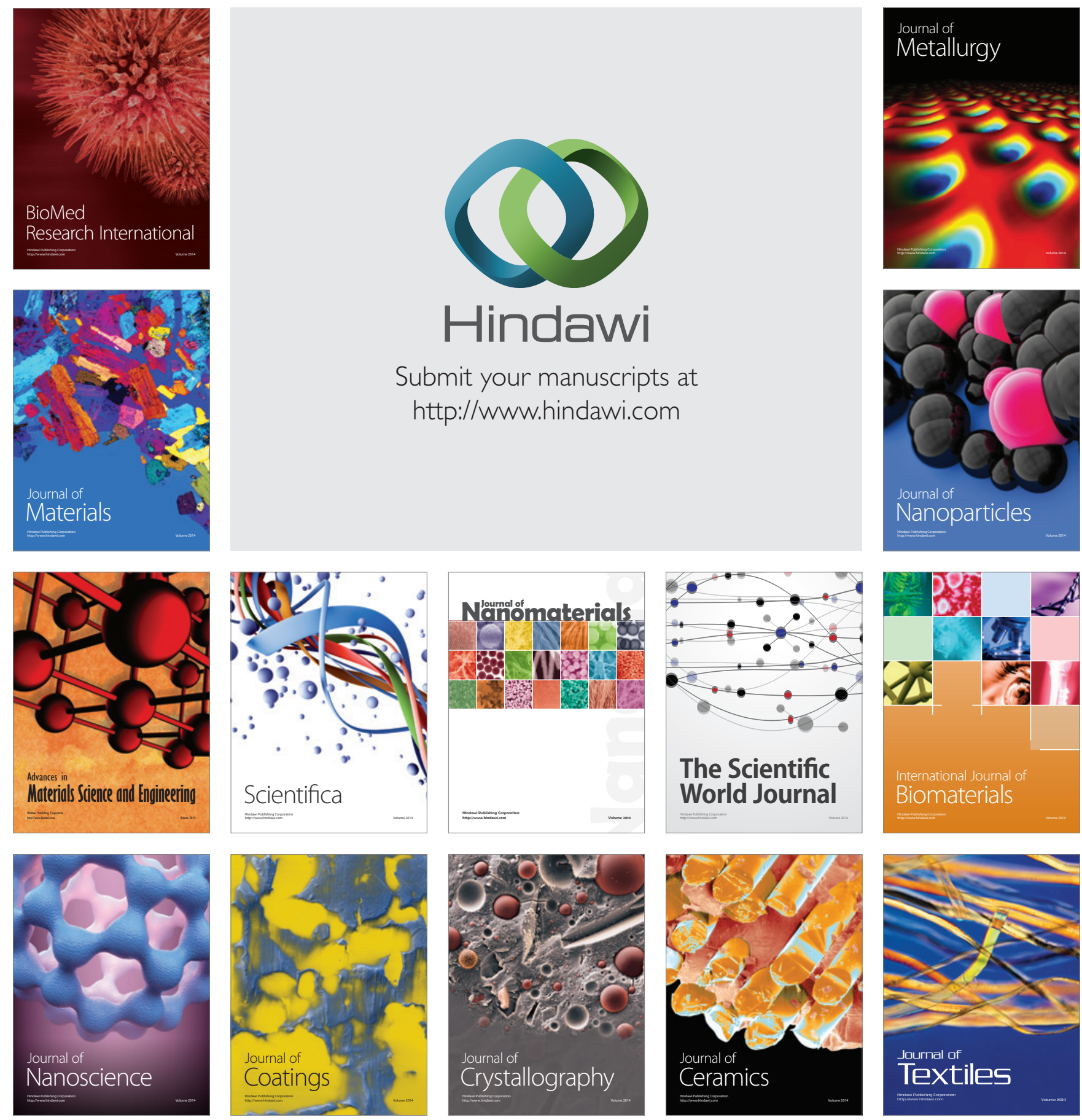